\title{
Der soziale Konflikt in der Regierungszeit der Unidad Popular
}

\section{Franz Hinkellammert}

Dem Programm, mit dem Allende die Unidad Popular in den Präsidentschaftswahlen von 1970 in Chile an die Regierung brachte, sah man nicht ohne weiteres an, wieviel Konfliktstoff es in sich barg. Es stellte sich als ein revolutionäres Programm vor, suchte aber seine Verwirklichung innerhalb des Rahmens der in Chile geltenden Gesetzlichkeit. In seiner vorsichtigen Formulierung sprach das Programm auch nicht davon, den Sozialismus aufbauen zu wollen. Es versprach vielmehr, Grundlagen für den Aufbau des Sozialismus zu schaffen. Die Entscheidung dariber, ob auf diesen Grundlagen der Sozialismus aufgebaut werden solle, würde daher frühestens erst bei den nächsten Präsidentschaftswahlen im Jahre 1976 gefallen sein. Die wirtschaft. lichen Maßnahmen, die diese Revolution innerhalb der Gesetzlichkeit ankündigte, bauten daher auch zum großen Teil auf bereits unter vorhergehenden Regierungen begonnenen Reformprojekten auf. Soweit diese Maßnahmen hierüber hinausgingen, bewegten sie sich in einer Richtung, in der - zumindest scheinbar - kein besonderer Widerstand der christdemokratischen Partei zu erwarten war, die die größte unter den Oppositionsparteien war.

Der Großteil dieser wirtschaftlichen Maßnahmen richtete sich gegen das chilenische Großbürgertum, die Großgrundbesitzer und das Auslandskapital. Die gegen den Großgrundbesitz gerichtete Agrarreform war bereits unter der Regierung Frei begonnen worden und sollte jetzt mit größerer Geschwindigkeit innerhalb des herrschenden Gesetzes zu Ende geführt werden. Soweit die Nationalisierung des Auslandskapitals die Kupferbergwerke betraf, so entsprach sie durchaus der Logik des unter den Christdemokraten gescheiterten Versuches, diese Unternehmen ohne Nationalisierung unter Kontrolle zu bringen. Am weitesten ging wohl der Plan der Regierung, die Großindustrie zu verstaatlichen, über die Ziele der christdemokratischen Partei hinaus. Aber er traf auch nicht auf irgendeinen prinzipiellen Widerstand, da ja selbst der christdemokratische Präsidentschaftskandidat Tomic in seiner Wahlkampagne 1970 Schritte in dieser Richtung angekündigt hatte.

Neben diesen die Eigentumsstruktur berührenden Maßnahmen stand eine weitere, die geradezu in einer absoluten Ubereinstimmung mit dem christdemokratischen Programm zu stehen schien. Es handelt sich um die Einkommensumverteilung zugunsten der niedrigsten Einkommen, die fast ausschließlich von den höchsten Einkommen getragen werden sollte. Ihr Ziel war, die unteren Einkommen soweit anzuheben, daß ein erträgliches Lebenshaltungsminimum für alle Chilenen gesichert sei und dabei die mittleren Einkommen nicht betroffen würden. In der gleichen Richtung hatten die Christdemokraten zwischen 1964 und 1970 bereits eine Einkommensumverteilung auf dem chilenischen Land durchgeführt und dabei auch relative Erfolge zu verzeichnen gehabt. Es schien fast selbstverständlich, daß sie sich einer ähnlichen Politik in der Stadt, für die sie durch die Promocion Popular auch schon Grundsteine gelegt hatten, nicht widersetzen würden. 
Die Revolution innerhalb der Gesetzlichkeit schien daher gleichzeitig eine Revolution mit Pasteten und Rotwein zu werden, in der der Wein an die Stelle des Blutes trat. Sie würde nur die Interessen einer sehr kleinen, ökonomisch starken Schicht treffen, ohne ihren Mitgliedern die Lebenschancen zu rauben.

Das Programm sprach zwar von einem Klassenkonflikt, hatte ihn aber in Wirklichkeit nicht vorgesehen. Der Übergang würde harmonisch sein, abgesehen von einigen überwindbaren Interessenkonflikten.

Entsprechend waren auch die Reaktionen auf das erste Jahr der Regierung der Unidad Popular. Einzig die Oberklasse und das Auslandskapital reagierten mit äußerster Entschiedenheit. Ein kurz vor dem Amtsantritt Allendes von ihnen inszenierter Putschversuch scheiterte, da er sich in einer absoluten Isolierung gegenüber fast dem gesamten Volk befand, obwohl er zur Ermordung des Oberbefehlshabers des Heeres, General Schneider, führte. Der Oberklasse und dem Auslandskapital blieb nur der Finanz- und Ersatzteilboykott, der sich allerdings erst nach einer längeren Periode auswirken konnte. Die Maßnahmen zur Veränderung der Wirtschaftsstruktur, die im Jahre 1971 begannen, fanden daher auch kaum Widerstand von seiten der Mittelklassen. Die Nationalisierung des Kupfers wurde einstimmig vom Parlament angenommen. Die Verstaatlichung der Privatbanken konnte mit der Unterstützung der Bankangestellten und des größten Teils der Öffentlichkeit rechnen. Die Großunternehmungen, die verstaatlicht wurden, fanden beim Kleinbürgertum der Händler und Kleinunternehmer nur geringen Widerhall. Die sehr schnelle Beendigung der Agrarreform innerhalb der beiden ersten Regierungsjahre stieß lediglich auf den Widerstand der besitzenden Schicht auf dem Lande. Allerdings handelte es sich hierbei bereits um eine zahlenmäßig größere Gruppe, die jedoch wenig Rückhalt in den Städten fand.

Ebenfalls konnte die Einkommensumverteilung zugunsten der unteren Einkommensschichten einige Erfolge zeigen. Die Arbeitslosigkeit sank rasch ab, und die Familieneinkommen der unteren Einkommensschichten wuchsen an. Das Jahr 1971 und die erste Hälfte des Jahres 1972 stellen daher wohl die besten Jahre dar, die diese Gruppen in der chilenischen Geschichte je erlebt haben.

Aber bereits im Jahre 1971 gab es beunruhigende Zeichen. Die Einkommensumschichtung zugunsten der unteren Einkommensschichten führten nicht zu einer wirklichen Konsumumschichtung. Die gesamte Unternehmerschaft - große und kleine Unternehmer - senkte ihre Investitionen um mehr als $66 \%$ und verwendete den nicht investierten Teil ihrer Einkommen zu Konsumzwecken. Daher stieg nicht nur der Konsum der unteren Einkommensschichten an, sondern ebenfalls der Konsum der mittleren und hohen Einkommen. Es trat damit von der Seite der Investitionen her ein Zustand ein, der nicht lange andauern konnte. Die Regierung nahm dieses Signal keineswegs so ernst, wie es war, und glaubte, daß die Investitionsbereitschaft dieser Unternehmer zum Großteil auf eine vorläufige Haltung des Abwartens zurückzuführen sei, die durch eine konstante Wirtschaftspolitik überwindbar sei.

Ebenfalls wurde sehr bald klar, daß dieser Wirtschaftsaufschwung durch einfache Nachfragestimulierung entstanden war und seine Möglichkeit im Grunde dar- 
auf beruhte, daß relativ hohe Devisenvorräte und Rohstoff- und Produktlager bestanden. Dies mußte um so mehr zu Schwierigkeiten führen, als der Finanz- und Ersatzteilboykott den Devisenzugang Chiles beschränkte und die notwendigen Importe verteuerte oder sogar unmöglich machte. Da der Ersatzteilboykott am schnellsten die Kupferproduktion traf, wirkte er sich seinerseits auch auf die chilenischen Exporterlöse aus.

Im Laufe des Jahres 1972 machte sich daher eine generelle Devisenknappheit bemerkbar, die zum Rückgang der Wachstumsraten der Produktion insgesamt und zum absoluten Produktionsrückgang und Stagnation in entscheidend wichtigen Bereichen - insbesondere in der Nahrungsmittelversorgung - führte. Die Nahrungsmittelversorgung ging sowohl deshalb zurïck, weil 1972 die Landwirtschaft stagnierte, als auch deshalb, weil die Nahrungsmittelimporte gedrosselt werden mußten.

Krisen dieser Art hatte die traditionelle chilenische Wirtschaft schon öfters durchgemacht, obwohl es sich diesmal um eine besonders schwere Krise handelte. Unter der christdemokratischen Regierung Frei hatte man ebenfalls den Versuch gemacht, die niedrigen Einkommen anzuheben. Dies führte ebenfalls zu einer besonderen Belastung der Nahrungsmittelversorgung. Man hielt diese Politik während der Jahre 1965 und 1966 durch, brach sie dann aber Ende 1966 ab und kehrte zu einer größeren Ungleichheit der Einkommen zurück, wobei der Umverteilungseffekt zugunsten niedriger Einkommen nur im Agrarsektor erhalten blieb. Die Jahre von 1967 bis 1970 waren daher durch eine Tendenz zu einer Erhöhung der Einkommensungleichheit und durch repressive Lohnpolitik gekennzeichnet.

Im Jahre 1972 war auch für die Unidad Popular dieser Punkt gekommen, an dem die Weiterführung der Einkommenspolitik mit der traditionellen Wirtschaftspolitik unvereinbar wurde. Die Stagnation der Nahrungsmittelversorgung und die relativ erhöhten unteren Einkommen, die einen besonders hohen Nachfrageanteil nach Nahrungsmitteln haben, führten zu einem relativ schnelleren Preisanstieg als dem Durchschnitt aller Preise entsprach. Der Mechanismus der freien Märkte machte daher ganz automatisch den Eink@mmensanstieg der unteren Einkommen wieder rückgängig.

Die Regierung der Unidad Popular stand daher im Jahre 1972 vor der Alternative, entweder die höheren Einkommen zu senken und die niedrigen Einkommen zu erhalten, oder aber die niedrigen Einkommen der Erhaltung der höheren Einkommen zu opfern. Gleichzeitig war offensichtlich, daß die traditionalen Marktmechanismen nur mit dieser zweiten Alternative zu vereinbaren waren, und daß in dieser Situation die Erhaltung der unteren Einkommen nur durch Außerkraftsetzung dieser Marktmechanismen, durch verschärfte Preiskontrollen und durch Lebensmittelrationierung erreicht werden konnte.

Vorhergehende Regierungen hatten in vergleichbaren Situationen grundsätzlich die Erhaltung und Erhöhung der höheren Einkommen zusammen mit der Erhaltung des Marktmechanismus vorgezogen. Die Regierung der Unidad Popular hingegen sah sich zu einer solchen Politik nicht in der Lage. Sie erklärte es zum Grundrecht jedes Menschen, eine minimale Versorgung mit lebenswichtigen Gütern und Dienstleistungen zu erhalten, so daß in einer Situation allgemeiner Knappheit sich 
die höheren Einkommen dieser grundsätzlichen Notwendigkeit anzupassen hätten.

Viel schneller noch als zu erwarten gewesen wäre, hatte sich so aus der Ein. kommensumverteilungspolitik ein die gesamte Gesellschaft durchziehender Konflikt gebildet. Er war gerade auf dem Gebiet entstanden, auf dem die Gemeinsamkeit zwischen Unidad Popular und christdemokratischer Partei am größten schien. Um ihre Einkommenspoltik durchführen zu können, sah sich die Unidad Popular zu stärkerer Intervention der Unternehmen, zur Kontrolle der Preise und in steigendem Umfang zur rationierten Güterverteilung gezwungen, während die Opposition die Erhaltung der Marktmechanismen über die Einkommensverteilung stellte und so implizit darum kämpfte, die niedrigen Einkommen der Erhaltung der höheren Einkommen zu opfern.

Dieser Basiskonflikt bricht im Jahre 1972 auf und gibt die Grundlage ab für die außerordentliche Zuspitzung der sozialen Kämpfe im Jahre 1973 und ihren Ausgang im Putsch vom 11.9.73. Er ist von Seiten der Unidad Popular nichts anderes als ein Kampf darum, die minimale Versorgung mit Gütern und Dienstleistungen zum Recht eines jeden Mitgliedes der Gesellschaft zu machen.

Die Regierung der Unidad Popular sielit sich auf den Standpunkt dieses Lebensrechts und weigert sich daher, es dem Funktionieren der traditionalen Marktwirtschaft und ihrer Mechanismen unterzuordnen. Sie versuchte hingegen, aus diesem Grundrecht abzuleiten, welche Eigentumsordnungen und welches Verteilungssystem und welche Arbeitsplatzsicherung diesem Grundrecht adäquat seien. Sozialismus wurde jetzt immer klarer als eine Sozialordnung aufgefaßst, die in Chile dieses Grundrecht in absehbarer und kurzer Zeit zu geben in der Lage war. Die sozialen Kämpfe selbst machten klar, daß das traditionale kapitalistische System Chiles - in welchen $\mathrm{Abwandlungen} \mathrm{auch} \mathrm{immer} \mathrm{-} \mathrm{dieses} \mathrm{Grundrecht} \mathrm{nicht} \mathrm{gewähren} \mathrm{konnte.}$

Indem aber dieser Konflikt ausbrach, hatte das chilenische Großbürgertum und das Auslandskapital erstmals die Möglichkeit, sich eine Massenbasis zu bilden. Diese Massenbasis ergab sich in jenen sozialen Schichten, für die die Anerkennung dieses Grundrechts einen Eingriff in ihre wirtschaftlichen Interessen bedeutete. Der erste Einbruch gelang ihnen bei den als aristokratisch geltenden Akademikerberufen, wie den Arzien, den Rechtsanwälten und den Ingenieuren. Vor allem die Ärztekammer verkündete sehr lautstark, daß die Ärzte gemäß ihrer , hohen sozialen Mission $^{66}$ und nicht gemäß3 der wirtschaftlichen Möglichkeiten des Landes bezahlt werden müßten. Zum erstenmal aber gelang auch, das Kleinbürgertum der Händle und kleineren Unternehmer zur Solidarisierung mit dem Großbürgertum zu bringen. Ebenfalls schlossen sich Teile der Staatsbürokratie dieser Bewegung an, die im Oktober $1972 \mathrm{zu}$ einem ersten, länger als einen Monat dauernden Unternehmerstreik. aufrief. Besonders zwei Gruppen hatten dabei großen Anteil, nämlich die Lastwagenbesitzer und die Ärzte. Insbesondere der Arztestreik - der von etwa $2 / 3$ der Ärzte befolgt wurde -, offenbarte, daß die Opposition zu jedem Mittel der Auseinandersetzung entschlossen war und daß von der Seite der Opposition nicht mehr in humanitären Kategorien gedacht wurde.

Ein Einbruch in die Aübeiterschaft gelang der Rechten nicht. Auch die christdemokratische Arbeiterschaft nahm am Streik nicht teil. Auf dem Land gab es 
isolierte Streikbewegungen ausgehend von christdemokratisch beherrschten Gewerkschaftsvertretungen, aber diese blieben in einer klaren Minderheit und brachen bald $a b$. Die Unternehmungen arbeiteten daher, während die Unternehmer streikten. Dennoch wurde das Land praktisch paralysiert, da nur ein sehr geringer Teil des Transportwesens noch funktionierte. Das Land war eindeutig in zwei Lager gespalten.

Dieser Oktoberstreik wurde abgebrochen, da sich zeigte, daß die Streitkräfte noch nicht zum Putsch bereit waren. Das Großbürgertum konnte zusammen mit den mobilisierten Mittelklassen zwar das Land paralysieren, konnte aber die Macht nicht ibernehmen.

Für die Unidad Popular aber galt etwas ähnliches. Da sie an der Regierung war, konnte sie auf ihrer Politik bestehen, hatte aber weder die Macht noch die Überzeugungskraft, sie auch durchzuführen. Trotz des Streikabbruchs im November 1972 war die Haltung der Mittelklassen eine Haltung des Boykotts, der durch den Finanaz- und Ersatzteilboykott von außen nur verstärkt wurde. Da aber die Regierung der Unidad Popular weder zurück noch vorwärts konnte, ergab sich im Jahre 1973 eine weitgehende Paralysierung des Landes. Die Opposition versuchte, diese Pattsituation durch die Abgeordnetenwahl im März $1973 \mathrm{zu}$ entscheiden. Es gelang ihr aber nicht, die verfassungsmäßige Mehrheit zu erreichen. Das Volk unterstützte die Parteien der Unidad Popular mit etwa $44 \%$ der Stimmen, während die Opposition zum Sturz des Präsidenten $66 \%$ der Parlamentssitze benötigte.

Unmittelbar nach den Wahlen ging daher die Opposition zur Gewaltanwen. dung vor. Es begann eine Attentatswelle gegen Menschen und Sachen, die bis zum 11. September nicht mehr unterbrochen wurde. Die den Putsch vorbereitenden Militärs unterstiitzten die Opposition mit Waffen und Sprengstoffen, die Polizei schritt nur beschränkt ein. Am 26. Juni riefen die Fuhnunternehmer aufs neue den Streik aus. Die Beteiligung der Lastwagenbesitzer war $\mathrm{zW}$ ar etwas geringer als im Oktober, aber zusammen mit der terroristischen Bewegung und der viel längeren Streikdauer ruinierte er die Wirtschaft in noch größerem Ausmaße.

Es waren wiederum die gleichen Gruppen wie im Oktoberstreik, die jetzt an diesen Streiks teilnahnen. Soweit die öfentlichen Angestellten Christdemokraten waren, beteiligten sie sich fast restlos - außer an den Tagen, an denen das Gehalt gezahlt wurde - am Streik. Die christdemokratische Arbeiterschaft nahm auch diesmal nicht am Streik teil. Einziger wichtiger Einbruch der Opposition in die Arbeiterschaft war der Streik der Kupferarbeiter von El Teniente gewesen, an dem sich der christdemokratische Teil der Arbeiterschaft dieses Kupferbergwerks beteiligte und der zu einer ganz außerordentlichen Devisenbelastung für ein Land führte, das unter dem Druck eines auswärtigen Finanzboykotts stand. Dieser Streik war aber bereits abgebrochen worden, bevor der zweite Fuhunternehmerstreik ausbrach.

Der Ärztestreik, der diesen Fuhrunternehmerstreik begieitete, war diesmal noch rücksichtsloser als im Oktober. Da auch diesmal sich nur etwa Zweidrittel der Ärzteschaft beteiligte, griffen die streikenden Ärzte zu Sabotagemaßnahmen, um den notdürftig aufrechterhaltenen Betrieb von Krankenhäusern und Unfallstationen 
zu unterbinden. Im September war schließlich die Regierung dem Terror gegen Gesunde und Kranke, dem Boykott von innen und von außen nicht mehr gewachsen. Als der Präsident Allende jetzt versuchte, durch den Ausruf eines Plebiszits die Pattsituation zu lösen, beeilten sich die Streitkräfte, den Putsch durchzufuhren. Am 11. September nahmen sie sich die Macht mit Gewalt und trieben jetzt den Terror auf die Spitze, der bereits viele Monate vor dem Putsch begonnen hatte.

Bei der Beurteilung dieser sozialen Kämpfe ist es von außerordentlicher Wichtigkeit, den tatsächlichen sozialen Konflikt richtig zu erkennen: Dabei braucht man nicht viele Worte über den Ausgangspunkt des Konflikts zu verlieren. Aus den Programmpunkten, die sich auf die Nationalisierung des ausländischen und inländischen Großkapitals, des Großgrundbesitzes und auf die Einkommensumverteilung beziehen, ergibt sich bereits eine klare Konfliktsituation zwischen Arbeiter- und Bauernschaft und dem Großbürgertum. Der Putschversuch vor der Regierungsübernahme durch Allende 1970 im Zusammenhang mit der Ermordung des Oberkommandierenden des chilenischen Heeres, General Schneider, und den Interventionsversuchen der ITT und des State Departments zeigen ebenfalls, daß dieses Großbürgertum diesen Konflikt mit allen Mitteln zu gewinnen versuchte.

Was zu erklären ist, ist vielmehr, wanum das Großbürgertum solche Massen der Bevölkerung auf seine Seite ziehen konnte, und warum diese Massen der Mittelklassen das Programm der Unidad Popular als eine solch tödliche Bedrohung aufzufassen begannen. Erst wenn dies erklärt ist, ist der grundlegende Konflikt dieser Zeit tatsächlich verstanden. Der Konflikt zwischen Arbeiter- und Bauernschaft auf der einen und dem Großbürgertum auf der anderen Seite mußte sich erst zu einem Konflikt zwischen den in der Unidad Popular organisierten Arbeitern, Bauern und Teilen der Mittelklasse auf der einen, und dem sich in der Opposition formierenden Zusammenschluß von Großbürgertum und Mehrheit der Mittelklasse auf der anderen Seite entwickeln um tatsächlich zu einer Polarisierung des gesamten chilenischen Volkes zu führen. Erst in dieser Form ist der Konflikt tatsächlich begriffen, wenn man außerdem verständlich machen kann, warum die Opposition schließlich zum Entschluß kam, die in der Unidad Popular organisierten Gruppen soweit physisch auszurotten, daß sie nie wieder die Möglichkeit zur Reorganisierung finden könnten.

Diese Entwicklung des Konflikts geht in den Jahren 1971 bis 1973 vor sich. Seine Massenbasis findet das ausländische und inländische Großbürgertum vorzüglich in zwei Richtungen. Eine Richtung ist die der sog. Professionales, also Arzte, Rechtsanwälte, Techniker, Ingenieure, Manager, hohe Beamte und im allgemeinen der Akademikerstand. Die andere Richtung bezieht sich auf das sog. Kleinbürgertum, also kleine und mittlere Unternehmer, Handel, privates Transportwesen.

Während die Entwicklung der Massenbasis des Großbürgertums in diesen zwei Richtungen vor sich geht, wird diese Entwicklung durch die Einkommensumverteilungs- und Vollbeschäftigungspolitik der Unidad Popular vermittelt. In dem Grade, in dem sich die Politik der Nationalisierungen und Enteignungen durch die Unidad Popular mit dieser Einkommensumverteilungs- und Beschäftigungspolitik verbindet, 
entwickeln sich diese Teile der Mittelkiasse zunehmend zu den Positionen des Grosbürgertums hin. Man wird also fragen müssen, in welcher Form eigentlich für diese Mittelklassen diese politische Richtung der Unidad Popular spürbar wird. Sie wird es als Umverteilungs- und Vollbeschäftigungspolitik.

Die Unverteilungspolitik scheint bei oberflächlicher Betrachturg ein primär quantitatives Problem zu sein. Die einen bekommen quantitativ mehr und die anderen quantitativ weniger. Aber es wird gleichzeitig insofern als qualitatives Problem empfunden, als die quantitative Einkommenshöhe immer mit der Frage der, gerechten ${ }^{6}$ Entlohnung verbunden ist. Eine Einkommenssenkung erscheint dann nicht mehr als ein quantitatives Weniger, sondern als eine Ungerechtigkeit und Verletzung der Menschenwürde.

Sofern daher die Umverteilung nicht nur die Kapitaleinkommen des Großbirgertums betraf, sondern auch die hohen Einkommen des Mittelstandes und insbesondere der Professionales, trat dieses qualitative Moment immer mehr in den Vordergrund. Für die Gruppe der Professionales gilt in einem ganz besonderen Maße, daß ihre Vorstellungen von ihrem, gerechten" Einkommen durch Demonstrationseffekt und Tradition vorbestimmt sind. Da sie einen hohen Grad internationaler Verbindurgen haben, messen sie das ihnen, zustehende" Einkommen an den internationalen Einkommensstandards ihrer Gruppe. Von daher nimmt man die Einkommensniveaus des Arztes, des Technikers, des Universitätsprofessors etc. als statisch bestimmte Niveaus, die in aller Welt - natülich kapitalistischer Welt - gelten und die als materielle Anerkennung ihrer ,sozialen Mission" verstanden werden. Diese Professionales fühlen sich daher von allen ihren Pflichten gegenuber der Gesellschaft entbunden, wenn sie materiell nicht dieser ihrer Mission entsprechend anerkannt werden. Die rein quantitativ erscheinende Einkommensumvertellung wird so zu einem qualitativen Problem sozial-explosiven Charakters. Der Professional fühlt sich in seiner Menschenwürde betrogen, und handelt entsprechend, wenn seine Konsum. möglichkeiten angegriffen werden.

Die Unidad Popular aber mußte, wenn sie das Land entwickeln wollte, diese Einkommen berïhren. Das chilenische Volkseinkommen ist so niedrig, daß die Bezahlung aller dieser Gruppen gemäß ihrer nach internationalen Siandards bemessenen ,sozialen Mission" für den Rest nur den Hunger ibrigläßt. Die Unidad Popular stellte daher diesem Prinzip der Bezahlung nach, sozialer Mission" die Bezahlung gemäß den wirtschaftlichen Möglichkeiten des landes entgegen. Diese höheren Einkommen sollten nur einen solchen Teil des Volkseinkommens in Anspruch nehmen können, daßs für jeden Chilenen die Sicherung seiner Grundbedürfnisse möglich wurde. Es ist daher klar, daß die Professionales die Politik der Unidad Popular als gegen die Menschenwürde gerichtet und materialistisch ansahen. Als dann nach dem Putsch vom 11. September die Würde der Arbeit wiederhergestellt wurde, hieß dies ebenfalls, daß für die Massen der Hunger wiederhergestellt wurde. Der Materialismus war ausgerottet und Ideen und Prinzipien wurden wieder geachtet.

Die Professionales aber fühlten sich nicht nur von der Einkommensumverteilung in ihrer monetären Form her bedroht. Mindestens ebensosehr reagierten sie 
gegen die mit der Umverteilung verbundene Vollbeschäftigungspolitik der Unidad Popular. Diese Reaktion hängt mit den Ansprüchen zusammen, die die Unidad Popular an die Leistungen der Professionales stellte. Diese Anspriiche hängen eng mit der Notwendigkeit einer Umstellung der Produktionsstruktur im Verlauf der Einkommensumverteilungs- und Vollbeschäftigungspolitik zusammen.

Damit ein Volkseinkommen umverteilt werden kann, muß es aus solchen Produkten bestehen, die ihrer physischen Beschaffenheit nach umverteilt werden können. Solange die Produkte relativ homogen und teilbar sind. stellt die Umverteilung -- was vor allem für Nahrungsmittel zutrifft - keine Probleme. Diese Produkte aber sind für die chilenische Industrie keineswegs typisch. Extremster Fall ist der der Automobilindustrie. Diese produziert etwa 20000 Personenwagen pro Jahr, die eben nur einer gleichen Zahl von Käufern zugute kommen können. Man kann eine solche Produktion nicht unterteilen und damit einer größeren Zahl zugänglich machen. Die Umvertejung kann hier nur zur Stagnation oder zur Produktionsumstellung auf Massentransportmittel wie Autobusse führen. Eine schnelle Entwicklung dieser Produktion würde sogar zu einer auf lange Sicht steigenden Ungleichheit der Einkornmen führen, da das Automobil nur von Personen mit entsprechenden Einkommen gekauft werden kanin und daher diese Einkommensgruppen mit der Geschwindigkeit der Automobilproduktion wachsen müssen. Dieses Problem der Umstrukturierung der Produkte trifft einen großen Teil der Industrieproduktion, wenn auch jeweils in unterschiedlichem Maße. So gilt es für die Möbelindustrie, die elektronische Industrie, das Bauwesen usw.

Es trifft die Endguitersphäre, weil ein Produkt produziert werden muß, das in seiner physischen Form unverteilbar ist. In einem analogen Sinne aber trifft es nicht nur die Endguterproduktion, sondern die gesamte Zwischengüterproduktion. Damit die Vollbeschäftigung möglich ist, muß das Produkt nicht nur umverteilbar sein, sondern auch mit solchen Technologien produziert werden, die alle Arbeitskräfte zu seiner Produktion erfordern. Nicht alle Technologien sind unter den chilenischen Bedingungen -- den Bedingungen eines unterentwickelten Landes - mit der Vollbeschäftigung der Arbeitskraft vereinbar. Vor allem die undiskriminierte Anwendung der entwickelsten Technologien der industrialisierten Länder zerstört mehr Arbeitsplätze als sie schafft, obwohl sie das Gesamtprodukề erhöht. Daher ist eine Abwägung nötig, die solche Technologien nur soweit fördert, wie sie zur Entwicklung der Arbeitsproduktivität einer vollbeschäftigten Arbeitskraft beitragen.

Dies hat aber insbesondere Auswirkungen auf die Tätigkeit der Professionales. Sollte Chile entwickelt werden, so mußte es gerade diejenige Konsumgüterproduktion vermeiden, deren Produkte in den entwickelten Ländern die höchste Attraktivität haben, und sie mußte Technologien entwickeln, die vom Standpunkt der entwickelten Länder aus eher rückständig und simpel erscheinen. Es mußten sich also nicht nur die Konsummöglichkeiten der Professionales ändern, sondern auch die Arbeitskriterien. Da ein Großteil der Produkte vereinfacht und in der Produktionstechnik simplifiziert werden mußte, die den Tendenzen der internationalen Standards konträr entgegengesetzt sind. Universitäten, Forschungsinstitute und Techniker durften sich daher nicht mehr kritiklos an den internationalen Standards 
ihrer jeweiligen Wissenschaften und den entwickeltsten Produktionsstätten orientieren.

Wissenschaftler und Techniker aber mußten dies gerade als Beschränkung ihrer Freiheit und Würde empfinden, solange sie ihren Erfolg daran messen, was in den international führenden Zeitschriften ihrer Wissenschaftszweige publizierbar ist und was auf internationalen Kongressen diskutiert wird. Praktisch aber klaffte eine Lücke zwischen diesen internationalen Standards und den Anforderungen, die das Interesse des Landes an diese Professionales stellte. Und dies galt nicht nur für die Techniker, sondern ebenfalls für einen weiten Bereich des Gesundheitswesens, das weitgehend auf den Bedürnissen einer kleinen, gut bezahlenden Oberschicht aufgebaut war und nun den Bedürnissen aller Chilenen dienen sollte.

Für die Professionales war daher sowohl von ihren Konsummöglichikeiten und von ihren Arbeitskriterien her eine Bedrohung gegeben. In ihrem BewuBtsein wurde diese Bedrohung - unter Mithilfe einer breiten Kormmunikationsmittelkampagne als ein Angriff gegen Freiheit und Menschenwürde interpretiert. Daher zogen sie in den Kampf gegen die Unidad Popular, um die Freiheit zu verteidigen. Niemals aber sagten sie, daß sie eine Freiheit verteidigten, deren Kehrseite das Elend der Mehrheit der Bevölkerung war. Aus der Interpretation ihrer Position als Verteidigung von Menschenwürde und Freiheit aber nahmen sie die Kraft, in dieser Auseinandersetzung jede Menschlichkeit zu vergessen.

Sie wollten aber nicht nur ihre Interessen verteidigen, sondern sie hatten Macht, es zu tun. Als Techniker, Ingenieure und Manager konnten sie, gestitzt auf den ausländischen Finanz- und Ersatzteilboykott, die Produktion erschweren. Allerdings gingen ihre Möglichkeiten auf diesem Gebiet nicht allzu weit, da innerhalb der Unternehmungen Kontrollmöglichkeiten der Arbeiterschaft bestanden und die Techniker zumindestens teilweise ersetzbar waren. Die wirklich gefährliche Macht entwickelte sich von seiten der Organisationen der Arzteschaft. Arbeiterstreiks und auch Techniker oder Unternehmerstreiks richten sich gegen die Giiterproduktion und sind daher in ihrem Effekt auf andere soziale Gruppen vermitteit. Die Arzteschaft hingegen entwickelte einen Streik, der sich direkt gegen Leib und Leben der anderen richtete. Da die Privatkliniken weiter funktionierten, richtete er sich direkt gegen das Leben der Arbeiter und Bauern. Er verbreitete wahre Panik in den Randbezirken Chiles. Schwangere Frauen begannen zu beten und Wallfahrien zu machen, damit die Geburt nicht in die Zeit eines Arztestreiks fallen möge. Die geringste Komplikation konnte dann den Tod bedeuten. Der Ärztestreik war der wahre Bote des Terrors.

Aber ein solcher Ärtestreik war nur möglich, wenn gleichzeitig andere Gruppen ihn unterstuitzten. Die Zahl der Arzte ist viel zu klein, um sich allein gegen das ganze Volk stellen zu können. Er wurde daher als diese Todeswaffe nur eingesetzt innerhalb massiver Streikbewegungen der Mittelklasse.

Diesen massiven Riickhalt konnten sich die Professionales selbst nicht geben. Die eigentliche Massenbasis gab ihnen vielmehr das Kleinbuirgertum, das auf seine Weise auf die Politik der Einkommensumverteilung und der Volibeschäftigung reagierte. Man muß daher fragen, wie dieses Kleinbürgertum durch diese Politik betrof. 
fen wurde.

Auf der einen Seite wurde es durch die Politik der Einkommensumverteilung betroffen. Diese führte zu einer relativen Erhöhung der niedrigeren Einkommen. Diese aber haben einen sehr starken Nachfrageanteil vor allem für Nahrungsmittel. Indem sich daher die niedrigeren Einkommen erhöhten, erhöhte sich überproportional die Nachfrage nach Nahrungsmitteln. Die Nahrungsmittelproduktion des Landes hingegen entwickelte sich nur langsam und war aus strukturellen und langfristigen Gründen eher stagnierend. Da man wegen der Devisenschwierigkeiten nur begrenzt auf Einfuhrerhöhungen zurückgreifen konnte, führte dies insbesondere auf dem Nahrungsmittelsektor zu Marktlücken. Daraus wiederum resultierten Tendenzen zu überproportionalen Preissteigerungen für Nahrungsmittel, die die Einkommensumverteilung wieder rückgängig zu machen drohten.

Sofern die Regierung auf diese Annullierung der Einkommensumverteilung durch die Inflation mit Hilfe von weiteren Erhöhungen der unteren Einkommensschichten reagierte, heizte sie offensichtlich nur die Inflation an, ohne ihr Ziel zu erreichen. Sie versuchte daher, strenge Preiskontrollen durchzuführen, um zu verhindern, daß die Preise auf das durch die Höhe der Gesamtnachfrage für Lebensmit. tel bedingte Niveau anstiegen. Dies führte jedoch sehr schnell zur Entwicklung von Parallel- und Schwarzmärkten, so daß bereits Ende 1972 ganz offensichtlich die Einkommenspolitik nur noch unter der Bedingung der Lebensmittelrationierung aufrechterhalten werden konnte. Die Macht der Regierung reichte jèdoch dafür nicht aus, so daß die Ansätze zu einem Rationierungssystem ganz außerordentlich lïckenhaft blieben. Auf der anderen Seite aber konnte die Regierung der Unidad Popular nur sehr schwer zurückweichen, wenn sie an ihrer Politik festhalten wollte, jedem Chilenen eine die Grundbedürfnisse garantierende Versorgung zu gewährleisten. Marktgleichgewicht bedeutete in einer solchen Situation, erhebliche Teile der Bevölkerung von der Lebensmittelversorgung auszuschließen.

Diese Situation hatte ihre Auswirkungen auf die Mittelklassen. Das Kleinbürgertum sieht seine Freiheit und seine Gerechtigkeit darin, in Ausnutzung der Marktchancen seine Gewinne zu machen. Hierin besteht seine Gemeinsamkeit mit dem Großbürgertum. Die Unidad Popular aber verlangte durch Rationierung und Preiskontrollen, bestimmte Marktchancen gerade nicht auszunützen und behandelte daher auf diesen Sektoren den ,freien ${ }^{66}$ Handel als illegal.

Die entstehenden Versorgungsschwierigkeiten aber hatten ihre Auswirkungen weit über den Kreis des Kleinbürgertums - insbesondere des Handels - hinaus und betrafen praktisch das ganze Volk. Arbeiter und Bauern interpretierten diese Schwierigkeiten eher als Úbergangsprobleme zu einer neuen, auf andere Weise geordneten Wirtschaft. Der Mittelstand hingegen einschließlich der Mehrheit des „,bürokratischen“ Mittelstandes, interpretierte die Situation - unter dem Einfluß der politischen Parteien und der Massenkommunikationsmittel der Rechten - als einen Ausbruch des Chaos im Konflikt mit Freiheit und Gerechtigkeit.

Das Kleinbürgertum war aber nicht nur durch die Probleme der Rationierung und Preiskontrollen betroffen, die es in seinen Verhaltenskriterien - Gewinnmaximierung auf der Basis von Marktgleichgewichten -- beschränkten. Diese gleichen 
Verhaltenskriterien wurden noch von einer anderen Seite her eingeengt. Dies hing damit zusammen, daß das Ziel der Unidad Popular, der Gesamtbevölkerung eine die Grundbedürfnisse garantierende Versorgung zu gewährleisten, nicht einfach nur durch eine Einkommensumverteilung zu erreichen war. Das Volkseinkommen selbst mußte wachsen. Das angezielte Wachstum aber mußte sich auf die soziale Infrastruktur und die Güter des allgemeinen Lebensbedarfs richten, und mußte das Wachstum der Güter des gehobenen Lebensbedarfs zurückstellen. Es ging also nicht um wirtschaftliches Wachstum überhaupt, sondern um ein ganz spezifisches wirtschaftliches Wachstum, in dessen Mittelpunkt die Güter des allgemeinen Lebensbedarfs zu stehen hatten.

Nun hatte aber bereits im Jahre 1971 eine Untersuchung der CORFO - chilenische Planungsbehörde für Industrieplanung - gezeigt, daß angesichts der aus den entwickelten Ländern fließenden neuen Technologien auf mittlere Sicht die höchsten Wachstumsraten und ebenfalls die höchsten Rentabilitäten bei der Konzentration auf das Wachstum von Gütern für den gehobenen Lebensbedarf zu erzielen sind und die Verwirklichung eines solchen Wachstumsmodells eine zunehmende Ungleichheit der Einkommen zur Folge hat (Industrielles Profil Chiles, CORFO 1971). Die Untersuchung der CORFO zeigte, daß bei einer mittleren Frist von etwa zehn Jahren die wirtschaftliche Wachstumsrate dann am höchsten sein würde, wenn die Automobilindustrie als führender Wachstumssektor ausgewählt würde. Dies hätte aber ein negatives Wachstum der Güter des allgemeinen Lebensbedarfs vorausgesetzt.

Derselbe Bericht der CORFO kam andererseits zum Ergebnis, daß die Wachstumsrate der Güter des allgemeinen Lebensbedarfs erheblich höher sein könne, wenn man sie in den Mittelpunkt der Wachstumspolitik stellte. In diesem Falle mußte aber die durchschnittliche Wachstumsrate der Gesamtwirtschaft niedriger sein als im Falle einer Konzentration auf die Automobilindustrie.

Die Unidad Popular konnte nur diese zweite Alternative wählen, da sie die einzige war, die eine Entwicklung des gesamten Landes garantierte. Die erste Alternative führte notwendig zur schnellen Entwicklung eines Teils und zur weiteren Unterentwicklung des anderen Teils. Sie wäre nichts weiter gewesen als die Fortsetzung eines schon klassisch gewordenen lateinamerikanischen Wirtschaftsmodells, das bereits in Mexiko und Brasilien sehr klar diese seine Tendenzen gezeigt hatte.

Aber diese Alternative der Unidad Popular bedeutete nicht nur die einfache Fortsetzung der Umverteilungspolitik auf dem Gebiete des Wirtschaftswachstums. Sie bedeutete gleichzeitig einen neuen Konflikt mit den Verhaltenskriterien des Bürgertums einschließlich des Kleinbürgertums der mittleren und kleinen Unternehmen. Sie mußte nämlich Investitionskriterien vertreten, die wiederum den Tendenzen der Gewinnmaximierung der Einzelunternehmen konträr waren. Vom Standpunkt der Einzelunternehmen aus gesehen, war die Investition in Produktionen des gehobenen Lebensbedarfs die rentabelste. Die Entwicklungspolitik des Landes aber mußte die Investitionen in Richtung der weniger rentablen, aber wichtigeren Guiter des einfachen Lebensbedarfs orientieren.

Auf diese Weise zeigte sich, daß es unmöglich ist, eine vorrangige Entwicklung 
der Güter des einfachen Lebensbedarfs dadurch zu erzeugen, daß man diese Güter besonders rentabel macht. Je rentabler sie sind, umso höher die dort gemachten Gewinne. Da die Gewinne aber - besonders in unterentwickelten Ländern - eine hohe Konsumneigung haben, führt die Gewinnerhöhung in der Produktion von Gütern des einfachen Lebensbedarfs zu einer Vergrößerung des Markts und damit der Rentabilität von Gütern des gehobenen Lebensbedarfs. Immer und notwendig schlägt sich daher die Erhöhung der Rentabilität in der Produktion von Gütern des einfachen Lebensbedarfs in eine noch stärkere Erhöhung der Rentabilität der Produktion von Gütern des gehobenen Lebensbedarfs um.

Die Produktionsanreize durch die Erhöhung der Rentabilität führten daher sowohl von der Seite der Technologien wie auch von der Seite der Entwicklung des inneren Marktes her ständig zu einem Wachstumsmodell, in dessen Mittelpunkt die Güter aes gehobenen Lebensbedarfes stehen.

Wollte also die Unidad Popular ihr Land tatsächlich entwickeln, so konnte sie es nur auf der Basis des Wachstums von Gütern des einfachen Lebensbedarfs tun und mußte daher Investitionskriterien einzuführen suchen, die gerade die rentabelsten Investitionen ausschlossen. Es kann daher nicht überraschen, daß sich das gesamte Bürgertum einschließlich des Kleinbürgertums durch eine solche Investitionspolitik bedroht fühlte.

Daß es Macht hatte, zeigte es ebenfalls durch seine Streikbewegungen. Diese seine Macht richtete sich gegen die Produktion von Gütern und war je nach Produktionszweigen sehr verschieden. Relativ schwache Auswirkungen hatten Streikbewegungen des Handelns, und die Streikversuche der mittleren Unternehmer scheiterten ganz generell an der Weigerung der Arbeiter, die Arbeit niederzulegen. Ihre volle Wucht aber entwickelte die Streikbewegung des Kleinbürgertums auf dem Gebiet des Transportwesens. Die Streiks der Lastwagenbesitzer konnten zwar die Produktion selbst nicht verhindern, konnten sie aber völlig unterminieren, indem sie den Austausch zwischen den Unternehmen weitgehend zum Erliegen brachten.

Dies ist die zusammenfassende Darstellung der grundlegenden Konfliktsituation, von der aus erst die dreijährige Geschichte der Unidad Popular verstanden werden kann. Es handelt sich um einen Konflikt zwischen dem Interesse einiger auf der einen und dem, Gesamtinteresse auf der anderen Seite, zwischen einem durch Produktion für Güter des gehobenen Lebensbedarfes oder Güter des allgemeinen Lebensbedarfes orientierten Wachstums, zwischen dem Recht einiger oder dem Recht aller, zwischen Überernährung einiger bei gleichzeitiger Unternernährung der Vielen oder einfacher Ernährung aller, zwischen dem Recht des Stärkeren oder des Schwächeren, zwischen wirtschaftlicher Irrationalität oder wirtschaftlicher Rationalität, zwischen Unterentwicklung oder Entwicklung, zwischen Kapitalismus oder Sozialismus, zwischen Bürgertum oder Arbeitern und Bauern, zwischen Ungerechtigkeit oder Gerechtigkeit. Es war der Kampf eines Volkes um die Gerechtigkeit als Recht.

Für die Unidad Popular war der Konflikt unvermeidbar. Sie hat eine Menge Fehler gemacht, aber die Tatsache, diesen Konflikt eingegangen zu sein, macht ihre Größe aus. Wäre sie ihn nicht eingegangen, hätte sie ihre Regierungszeit wahrschein- 
lich überlebt, aber sie wäre vom chilenischen Bürgertum sterilisiert worden, wie dies mit den zahllosen reformistischen Bewegungen vor ihr geschehen ist. Dadurch, daß sie den Konflikt bei Risiko ihres eigenen Unterganges aufgenommen hat, wies sie dem chilenischen Volk das Projekt einer neuen Freiheit, das mit dem Untergang der Unidad Popular keineswegs mitgestorben ist. Es lebt gerade durch den Tod der unzähligen Ermordeten.

Das Bürgertum reagierte mit einem doppelten $\mathrm{Haß}$, weil seine eigenen Vorstellungen von Menschenwürde und Freiheit sich dem chilenischen Volk als die Präsenz von Elend und Unterdruickung offenbarten, und weil im chilenischen Volk eine neue Idee von Freiheit und Menschenwürde entstand, die die Interessen dieses Volkes selbst in den Mittelpunkt des gesellschaftlichen Lebens silten. Das chilenische Bürgertum entschloß sich daher vom Moment der Regierungsübernahme durch die Unidad Popular an dazu, diese mit allen Mitteln zu zerschlagen und hatte daher keinen anderen Plan als den, die ganze Masse des Mittelstandes hierfür zu mobilisieren.

Die Form, in der das Bürgertum und die Parteien der Opposition diesen Konflikt ins Auge faßten, schloßs von vornherein die Bereitschaft aus, die Verfassungsmäßigkeit oder Gesetzlichkeit von Aktionen zur Basis der Konfliktlösung zu machen. Das Bürgertum wollte den Konflikt für alle Zeiten für sich entscheiden, mit oder ohne Verfassungsmäßigkeit. Da die Verfassung eine formale, bürgerliche Verfassung war, enthielt sie keine ausdrückliche Option, weder für noch gegen das Bürgertum. Sie enthielt lediglich formale Normen der Konfliktlösung, die aus dem Mehrheitskriterium abgeleitet waren. Da aber die verfassungsmäßige Mehrheit auf der Seite der Unidad stand und bis zum letzten Regierungstag der Unidad Popular dort verblieb, konnte das Bürgertum überhaupt nur hoffen, den Konflikt durch Verfassungsbruch für sich entscheiden zu können. In dem Grade, in dem es gegen die Unidad Popular arbeitete, arbeitete es daher an der Vorbereitung dieses Verfassungsbruchs, da es sowohl im Besitz der Waffen als auch der ökonomischen Macht war.

Die Unidad Popular hatte zwar das Recht auf ihrer Seite samt Verfassung und Gesetzlichkeit, aber sie hatte kaum Waffen und keine ökonomische Macht. Sie konnte sich nur auf ihre verfassungsmäßige Mehrheit stützen und war daher einem Bürgertum gegenüber wehrlos, für das die Verfassung längst ein Fetzen Papier war, das im äußersten Fall dazu diente, demagogische Vorwände zu liefern. Viel zu spät wurde es der Unidad Popular klar, ein wie großer Unterschied zwischen der Innehabung der Regierung und der tatsächlichen Macht besteht. Die Macht zu haben, hätte für die Unidad Popular bedeutet, fähig zu sein, ihr Regierungsprogramm durchzusetzen. Auf Grund der Verfassung stand ihr diese Macht zu, aber diese Machtfrage wurde vom Bürgertum nicht auf der Basis der Verfassung gestellt. Das Bürgertum war sich völlig klar darüber, daß die Macht aus den Gewehrläufen kommt und bereitete sich daher darauf vor, sie sich mit dem Gewehr zu nehmen. So machte es weniger Fehler, obwohl es im Unrecht war. 